\title{
Do not sweat it: we test while you rest and other updates on recent autonomic research
}

\author{
Mitchell G. Miglis ${ }^{1} \cdot$ Nicholas Larsen $^{1} \cdot$ Srikanth Muppidi ${ }^{1}$
}

Received: 4 May 2021 / Accepted: 4 May 2021 / Published online: 12 May 2021

(c) Springer-Verlag GmbH Germany, part of Springer Nature 2021

\section{Do not sweat it: we test while you rest}

The human body sweats constantly to regulate core body temperature, a process that also occurs at rest and during sleep. At-rest sweat is a much better indicator of underlying body physiology than exercise-, thermal-, or chemically induced sweat. Thus, being able to collect at-rest sweat could provide a valuable tool to measure sympathetic nervous system activity. However, at-rest thermoregulatory sweat is secreted at much lower rates than during exercise, making it challenging to collect and analyze. In their recent study Nyein et al. report on a wearable patch that can continuously measure thermoregulatory sweat at-rest [1]. The patch design incorporates microfluidics to combat evaporation and enable selective monitoring of secretion rates. Hydrophilic filters allow for a rapid uptake of sweat into sensing channels. The patch also contains electrochemical sensors for $\mathrm{Ph}$ and levodopa monitoring. The patch's small size allows for versatile body placement into body areas such as fingertips.

Normal sweating ranges from a few $\mathrm{nl} \mathrm{min} \mathrm{m}^{-1} \mathrm{~cm}^{-2}$ to 100's of $\mathrm{nl} \mathrm{min}^{-1} \mathrm{~cm}^{-2}$ [2]. Using the patch, the authors were able to detect flow rates as low as $2 \mathrm{nl} \mathrm{min}^{-1} \mathrm{~cm}^{-2}$. To test the feasibility of the micro collector, the authors had healthy volunteers wear the patch for $24 \mathrm{~h}$ on seven different body sites and were able to collect average sweat rates at all sites, with the highest sweat rate being on the fingers. The majority of the measured sweat rates were slightly lower than reported in the literature, which was attributed to lower environmental temperature and humidity in the laboratory. To show that the results were reproducible, a volunteer wore two patches on adjacent locations on the thigh while sleeping. The two locations had nearly identical sweat rates showing good reproducibility. Next, the authors performed sensor

Srikanth Muppidi

muppidis@stanford.edu

1 Department of Neurology, Stanford Medical Center, 213

Quarry Road, 2nd Floor, Palo Alto, CA 94304, USA characterizations (for chloride, $\mathrm{Ph}$, and levodopa) and found that the sensors had high sensitivity within the physiologic range. The authors then tested the patch sensors and were able to show that the sensors accurately calculated sweat rate when compared to the known input rate of a commercial pump system.

The author then performed a series of experiments to determine patch performance. The patch was placed on the wrist of a healthy volunteer along with a heart rate monitor for $6 \mathrm{~h}$. During sedentary periods the heart rate and sweat rate remained low, while intervals of activity such as walking caused both to rise and subsequently fall. The authors then placed the patch on two healthy volunteers for $24 \mathrm{~h}$ and sweat rate was measured during various activities including eating, public speaking, walking, sleeping, and while sedentary. During stressful events both heart rate and sweat rate increased. These results demonstrated that the patch could measure the body's routine sweat response during normal activities over full-day periods and help identify physiological deviating states such as stress. Next, the authors had a diabetic patient wear a finger patch during insulin injection. Glucose was initially high and rapidly dropped after insulin injection. While insulin dropped sweat rate increased. Once blood glucose level dropped below 90 there was a dramatic increase in sweat rate, while the heart rate was unaffected. These results demonstrated that the patch can assist in the identification of hypoglycemia and help in the treatment of diabetic patients. Finally, healthy volunteers consumed broad beans containing levodopa. Levodopa was detected in sweat about $20 \mathrm{~min}$ after initial intake, peaked $30 \mathrm{~min}$ after, and then slowly decreased. Levodopa concentration in sweat increased with increasing doses of the drug. The authors concluded that monitoring sweat levodopa may be a promising way to keep track of blood levodopa to assist patients in managing the motor symptoms of Parkinson disease.

This sweat patch enables near-real-time continuous monitoring of sweat rate and composition both at rest and with activity. The authors demonstrated how the patch has the 
potential to assist in detecting health conditions such as hypoglycemia, psychological stress, and blood levodopa levels. We certainly see the potential for the patch to be a useful diagnostic tool in autonomic medicine. Because the patch measures physiological sweating, it evaluates the integrity of both the central and peripheral sympathetic sudomotor pathways. There is potential for the patch to assist in the early detection of small fiber neuropathy, with higher sensitivity and better ease of use than conventional techniques. Furthermore, the patch could prove beneficial in monitoring treatment response in small fiber neuropathies over time. Future studies should determine the patch's reliability when used on a larger scale outside of a controlled environment. A major limitation of the patch is that sweat rate at rest can be confounded by parameters such as temperature and psychological stress. Nevertheless, we believe that the patch's ability to accurately and continuously measure sweat at-rest has great potential as a wearable device in the field of autonomic medicine.

\section{Towards a greater understanding of autonomic neurons in the spinal cord}

In our first editorial for this section of Clinical Autonomic Research, we reviewed the development of novel ways to analyze spinal autonomic neurons and reviewed an article by Espinosa-Medina et al., which proposed reclassification of sacral autonomic outflow as sympathetic based on genetic analysis of these neurons [3]. This raised many important and controversial questions on how autonomic neurons are classified (genetic, anatomical, physiological, or neurochemical) and led to a spirited discussion with editorial/reviews in this journal $[4,5]$. More recent work by our colleagues in genetics, while not as controversial, still is very important in moving the autonomic field forward.

In a recent publication in Nature Neuroscience [6], Blum et al. performed single-nucleus RNA sequencing on 43,890 neurons from the adult mouse with aims to elucidate the diversity of motor neurons in the spinal cords. To identify different types of neurons, researchers used genetic markers to separate autonomic from somatic motor neurons. Autonomic neurons were identified by their expression of neuronal nitric oxide synthases and were further clustered into 16 subtypes, with some clusters exhibiting a clear enrichment in the sacral spinal cord. The researchers then selected several genes enriched in these clusters and performed insitu hybridization on spinal cord segments. This analysis highlights some of the organizational logic of autonomic neurons. For example, clusters with rxfp1 gene (relaxin family peptide) were expressed in the sacral spinal cord, which correlates with male and female sexual function. Additional examples include clusters of autonomic neurons which express Neurotensin (Nts) are present throughout the thoracic and sacral lateral autonomic columns. The peptide Neurotensin is a strong inhibitor of sympathetic function, therefore, this finding suggests Nts gene expression is likely a key feature of these autonomic neurons. Finally, additional analysis looked for differentially expressed genes for neuropeptides, hormones and monoaminergic signaling pathways relevant to autonomic neurons. The researchers found genes ranging from serotonergic, adrenergic, hormone signaling and neuropeptides. The remainder of the analysis in this research study was focused on the spinal motor neurons.

Overall, these new findings suggest that the organization of autonomic neurons in the spinal cord is likely based on neuropeptide and hormonal receptor expression on these neurons. This analysis also raises the possibility that future treatment of autonomic diseases may involve selective targeting of specific autonomic neurons based on their genetic expression, thus providing precision medicine for complex autonomic disorders.

\section{To salt or not to salt in POTS: what is the evidence?}

Treatment of disorders of orthostatic intolerance such as orthostatic hypotension $(\mathrm{OH})$ and postural tachycardia syndrome (POTS) frequently involve non-pharmacological measures such as fluid and salt to increase blood volume, despite limited evidence to support these recommendations. In their publication in the Journal of the American College of Cardiology [7], Garland et al. performed one of the few clinical trials assessing the effect of dietary salt intake on symptom burden in adult POTS patients, as well as orthostatic tachycardia and other hemodynamic measures.

The authors used a randomized crossover design to compare orthostatic changes in standing heart rate (HR), as well as the maximum standing HR in 14 female POTS patients (age $35 \pm 8$ years), randomized to a low sodium $(10 \mathrm{mEq}$ sodium/day) or high sodium (300 $\mathrm{mEq}$ sodium/day) diet. A group of 13 age-matched female controls $(31 \pm 6$ years) were selected as a comparison group. All participants underwent supine and standing and plasma norepinephrine (NE) measurements, measures of blood volume with a tracerdilution technique, and 24-h urine electrolyte assessments. Participants were fed a low-monoamine, caffeine-free diet with $150 \mathrm{mEq}$ sodium on day 1 , then randomized to low or high sodium diets for 6 days, with regular urinary sodium measurements to confirm compliance. In an elegant study design, the authors accounted for hormonal effects by timing the intervention and measurement period to the same menstrual cycle phase for all participants. Water intake was not regulated. On day 7, participants underwent orthostatic stand testing. After $60 \mathrm{~min}$ of supine rest, participants stood for up 
to 30 min standing (as tolerated). Blood pressure (BP) and HR were measured continuously, and supine and standing $\mathrm{NE}$ levels were drawn from an indwelling IV catheter. The authors also performed laboratory measurements including plasma renin and aldosterone, plasma volume, and 24-h urine electrolyte assessments. Orthostatic symptoms were assessed with the Vanderbilt Orthostatic Symptoms Scale (VOSS).

Compared to the low sodium diet, the high sodium diet resulted in smaller orthostatic changes in HR in POTS patients (46 bpm [IQR 32-55 bpm] vs. $60 \mathrm{bpm}$ [IQR 55-64 bpm], $p=0.001$ ), however, there was no significant change in orthostatic BPs. Supine NE in POTS patients did not differ between high and low sodium diets, however, standing plasma NE was lower on the high salt diet $(753 \mathrm{pg} /$ $\mathrm{ml}$ [IQR 498-919 pg/ml] vs. 959 pg/ml [IQR 736-1,161 pg/ $\mathrm{ml}, p=0.017)$. Both supine and standing plasma renin and aldosterone were lower with a high sodium diet, as expected. Interestingly, there was no significant difference in symptom burden scores between POTS patients on low and high sodium diets, though the authors note that there was a nonsignificant trend towards lower symptom burden score in those on the high sodium diet. Compared to controls, POTS patients had a greater change in orthostatic HR and standing NE levels. On the low sodium diet, plasma volume in POTS patients was on average $11 \%$ less than expected for each individual's sex, height, and weight, however, this was reduced to $<1 \%$ on the high sodium diet, while symptom burden and orthostatic tachycardia remained, suggesting that volume depletion alone is unlikely a mechanistic explanation of the disease.

The limitations of this study include its small sample size, which may have been underpowered to detect changes in symptom burden, as well as the fact that fluid intake was not controlled for (it is unclear if medications were held during the trial). Nonetheless, we applaud the authors for bringing scientific rigor to a standard of care treatment that is assumed to be efficacious, and we look forward to more evidence-based therapies for patients with POTS.

Funding None.

\section{Declarations}

Conflict of interests None.

\section{References}

1. Nyein HYY, Bariya M, Tran B et al (2021) A wearable patch for continuous analysis of thermoregulatory sweat at rest. Nat Commun 12:1-13

2. Sonner Z, Wilder E, Heikenfeld J et al (2015) The microfluidics of the eccrine sweat gland, including biomarker partitioning, transport, and biosensing implications. Biomicrofluidics 9:31301

3. Espinosa-Medina I, Saha O, Boismoreau F et al (2016) The sacral autonomic outflow is sympathetic. Science (80-) 354:893-897 [online serial]

4. Espinosa-Medina I, Saha O, Boismoreau F, Brunet JF (2018) The "sacral parasympathetic": ontogeny and anatomy of a myth. Clin Auton Res 28:13-21

5. Jänig W, McLachlan EM, Neuhuber WL (2018) The sacral autonomic outflow: against premature oversimplification. Clin Auton Res 28:5-6

6. Blum JA, Klemm S, Shadrach JL et al (2021) Single-cell transcriptomic analysis of the adult mouse spinal cord reveals molecular diversity of autonomic and skeletal motor neurons. Nat Neurosci Nature Research 24:572-583

7. Garland EM, Gamboa A, Nwazue VC et al (2021) Effect of high dietary sodium intake in patients with postural tachycardia syndrome. J Am Coll Cardiol 77:2174-2184 